\title{
Study on resistance characteristics of submarine near water surface
}

\author{
Jiabao Chen, Bangjun Lv*, Likun Peng, and Bin Huang \\ College of Power Engineering, Naval University of Engineering, Wuhan 430033, China
}

\begin{abstract}
The submarine is usually affected by free surface and the navigation resistance increases when sailing near the surface. In order to study the specific resistance characteristics of submarine sailing near the surface, the SUBOFF with appendages was taken as the research object, and the calculation model was built based on Star CCM+ fluid simulation software, and the resistance coefficients under different submarine depths and speeds were calculated. Through comparative analysis, the influence of the depth and speed of the submarine on the resistance components was obtained, and the cause of the formation was analyzed. The results show that the influence of the depth of submarine on friction resistance coefficient is small in general. With the increase of the depth of the submarine, the pressure resistance coefficient decreases, and the wave amplitude decreases. The shear wave of Kelvin wave system is more obvious and the effect of scattering is weakened, which is of great significance for the study of submarine concealment. With the increase of speed, friction resistance coefficient decreases, the overall change trend of pressure resistance coefficient is first increased and then decreases. The interference effect between free surface and hull increases first and then decreases at each depth. The wave shape changes and resistance results mutually confirm. The free surface mainly generates waves by interacting with the hull, which affects the resistance characteristics of the submarine. The interference effect is greatly affected by the depth and speed of the submarine.
\end{abstract}

Keywords: free surface, resistance, diving depth, wave-making.

\section{Introduction}

As an important weapon to stabilize coastal defense, modern submarines have the characteristics of large combat radius, strong assault power and good concealment. Their concealment is mainly realized by diving in tens or even hundreds of meters of water depth. According to different power, submarines can be divided into conventional submarines and nuclear submarines. Today's mainstream conventional submarines usually use diesel electric power, which can be generated by diesel engine in the state of water or underwater ventilation pipe, stored in the battery, and then use the electric energy of the battery to sail

\footnotetext{
* Corresponding author: 2696496643@.qq.com
} 
underwater. Due to the need of concealment, after sailing underwater for a period of time, the conventional submarine generally floats to the underwater vent pipe and starts the diesel engine for charging. At this time, the submarine is sailing near the water surface. In addition to supplementing electric energy, the submarine usually needs to complete tactical maneuvers such as missile launch and observing the sea surface with periscope in this state. Compared with large-depth diving, when the submarine is sailing near the water surface, the free surface will have a great impact on its navigation performance.

At present, most scholars' research on the resistance characteristics of submarines mainly focuses on deep diving conditions, and only a few scholars have studied the near surface navigation of submarines. The main research method is to use CFD (Computational Fluid Dynamics) software such as fluent, CFX and Star CCM + to numerically simulate the flow around the submersible sailing near the water surface. Nan Zhang ${ }^{[1-2]}$ and others applied CFD software to carry out numerical simulation of submarine near surface navigation, mainly studied the flow field characteristics around submarine near surface and the influence of free surface on boat / propeller interference characteristics, and considered the influence of diving depth on resistance, but there was less analysis on the influence of speed; Jia $\mathrm{Li}^{[3]}$ studied ellipsoids and submarines sailing directly near the water surface, and analyzed the influence of submerged bodies at different depths on the free surface, but the research on the free surface waveform was not in-depth, and the variation of wave amplitude was not introduced in detail; Yunming Qiu ${ }^{[4]}$ carried out near surface numerical simulation of SUBOFF bare hull, calculated the wave making of free surface, and focused on the waveform characteristics at different speeds. Whether the conclusion is applicable to submarines with appendages remains to be verified; Wilson ${ }^{[5]}$ used CFX software to carry out hydrodynamic performance analysis of submarines under different depths and speeds with SUBOFF bare hull model as the research object, but only $1.556 \mathrm{~m}$ bare hull was selected. The applicability of the conclusion to model boats with appendages and larger scales needs to be further studied.

Based on the research of the above scholars, for the SUBOFF model with appendages, this study uses Star CCM + software to numerically analyze the resistance characteristics of near surface navigation, calculate the dimensionless friction resistance, pressure resistance and total resistance coefficient under different depths and speeds, focus on the impact of depths and speeds on each resistance component, and analyze the waveform characteristics. So as to further clarify the specific influence of free liquid on the resistance characteristics of submarine.

\section{Calculation model}

\subsection{Research object}

The research object is the SUBOFF model with full appendage published by Taylor Ship Research Center (DTRC), and its main dimensions and main parameters are shown in Table 1.

Table 1. Main parameters of SUBOFF.

\begin{tabular}{lc}
\hline Parameter & Numerical value \\
\hline Hull length $\mathrm{L} / \mathrm{m}$ & 4.356 \\
Hull breadth $\mathrm{B} / \mathrm{m}$ & 0.508 \\
Wet surface area S/m $/ \mathrm{m}^{2}$ & 6.348 \\
Underwaterdrainage volume $\nabla / \mathrm{m}^{3}$ & 0.706 \\
\hline
\end{tabular}




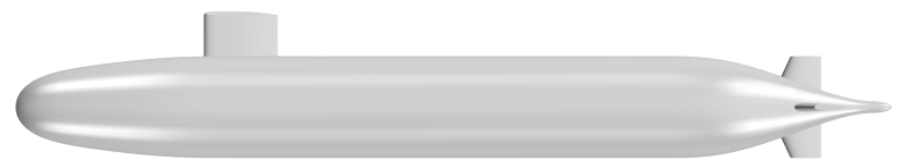

Fig. 1. SUBOFF geometric model.

\subsection{Calculation domain settings}

Since the SUBOFF model is symmetrical about the longitudinal section in the middle of the hull and the motion mode of the hull is fixed depth direct navigation, in order to save calculation resources, half of the calculation domain can be used for calculation, as shown in Figure 2. The calculation domain is roughly $5 \mathrm{~L} \times 2 \mathrm{~L} \times 3 \mathrm{~L}$ square domain in length, width and height, the boundary conditions are set as follows:

1) One time the length of the boat from the bow, and the boundary condition is set as the speed entrance;

2) 3 times the length from the stern, and the boundary condition is set as the pressure outlet;

3) The upper and lower boundaries of the basin are set as velocity inlets;

4) The left and right boundaries of the basin are set as symmetrical planes;

5) The hull surface is set as a non slip wall.

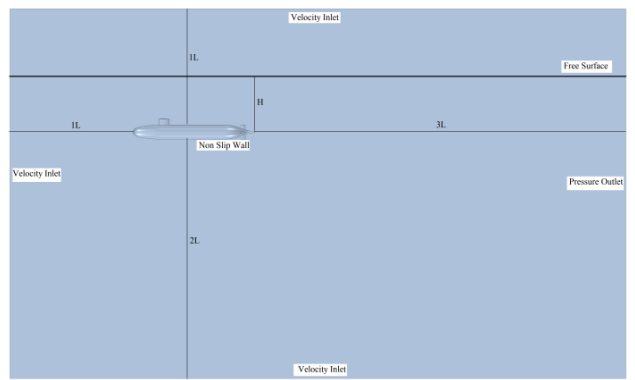

(a) Side view of computational domain

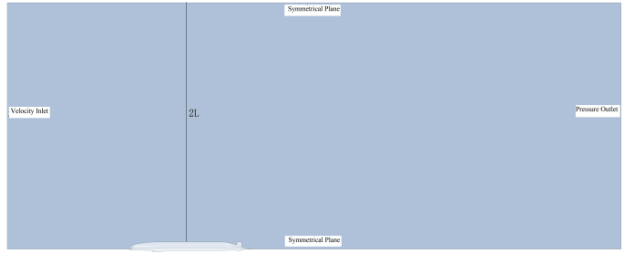

(b) Top view of computational domain

Fig. 2. Calculation domain and boundary conditions.

\subsection{Meshing}

Star CCM + unique trimmer mesh is adopted for mesh generation, which belongs to adaptive mesh. It can automatically reconstruct and repair the surface of the hull to improve the mesh quality. It can also improve the mesh generation accuracy of complex models by means of surface control, prism layer and mesh encryption. For hydrodynamic calculation, its calculation accuracy is comparable to that of structured grid ${ }^{[6]}$. The free surface needs to be considered when calculating the resistance of the hull sailing near the water surface. Due to the two-phase flow and the disturbance of the hull, the free surface flow is more complex. In order to better capture the free surface, it is also necessary to mesh the area near the free surface.

The basic grid size of the calculation model is $0.05 \mathrm{~m}$, the total number of grids is 1544000 , the prism layer is set as 5 layers, and the total thickness of the prism layer is $0.02 \mathrm{M}$. The meshing results are shown in Figure 3. 


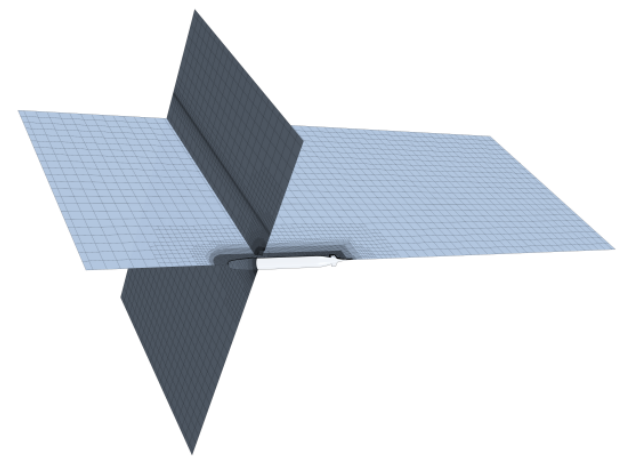

(a) Computational domain grid

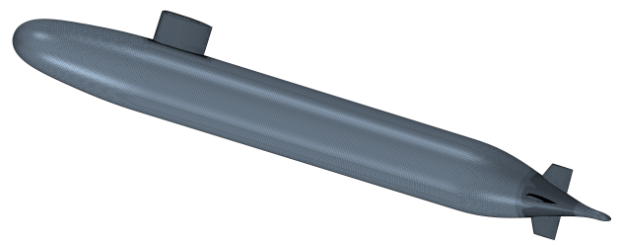

(b) SUBOFF face mesh

Fig. 3. Calculation domain and hull meshing.

\section{Calculation method}

\subsection{Governing equations}

The governing equations of the problem studied in this paper are the continuity equation of incompressible fluid flow and RANS equation ${ }^{[7]}$ :

$$
\begin{gathered}
\frac{\partial \overline{u_{i}}}{\partial x_{i}}=0 \\
\rho \frac{\partial \overline{u_{i}}}{\partial t}+\rho \overline{u_{j}} \frac{\partial \overline{u_{i}}}{\partial x_{j}}=\rho \overline{F_{i}}-\frac{\partial \bar{p}}{\partial x_{i}}+\frac{\partial}{\partial x_{j}}\left(\mu \frac{\partial \overline{u_{i}}}{\partial x_{j}}-\rho \overline{u_{i}^{\prime} u_{j}^{\prime}}\right)
\end{gathered}
$$

Among them, $\bar{u}_{i}$ is the time average velocity, $u_{i}^{\prime}$ is the pulsating velocity, and the related term of pulsating velocity $\rho \overline{u_{i}^{\prime} u_{j}^{\prime}}$ is called Reynolds stress.

In the numerical calculation of this study, the convection term adopts the second-order upwind difference and the dissipation term adopts the central difference. The simple method is used for pressure velocity coupling, and the time step is $0.04 \mathrm{~s}$.

\subsection{Turbulence model}

RNG $k-\varepsilon$ is used as the turbulence model in this paper.Turbulence model has good simulation ability.

The equation of turbulent kinetic energy $k$ is:

$$
\frac{\partial}{\partial t}(\rho k)+\frac{\partial}{\partial x_{i}}\left(\rho k u_{i}\right)=\frac{\partial}{\partial x_{j}}\left[\alpha_{k} \mu_{e f f} \frac{\partial k}{\partial x_{j}}\right]+G_{k}+G_{b}-\rho \varepsilon-Y_{M}+S_{k}
$$

The equation of turbulent dissipation rate $\varepsilon$ is:

$$
\frac{\partial}{\partial t}(\rho \varepsilon)+\frac{\partial}{\partial x_{i}}\left(\rho \varepsilon u_{i}\right)=\frac{\partial}{\partial x_{j}}\left[\alpha_{\varepsilon} \mu_{e f f} \frac{\partial \varepsilon}{\partial x_{j}}\right]+C_{1 \varepsilon} \frac{\varepsilon}{k}\left(G_{k}+C_{3 \varepsilon} G_{b}\right)-C_{2 \varepsilon} \rho \frac{\varepsilon^{2}}{k}-R_{\varepsilon}+S_{\varepsilon}
$$

where $C_{1 \varepsilon}=1.44, C_{2 \varepsilon}=1.9$. 


\subsection{VOF method}

In this study, the flow around the free surface is simulated by VOF (volume of fluid) method. The VOF method captures the free surface by calculating the volume share of the fluid in the grid cell. For pure air, the volume fraction of its grid element is 0; For pure water, the volume fraction of the grid element is 1 . The control volume unit of free surface contains air and water, the volume fraction of air is, and the volume fraction of water is, then the volume fraction transport equation of water is:

$$
\frac{\partial \alpha_{w}}{\partial t}+V \bullet \nabla \alpha_{w}=0
$$

Since the free surface control body unit is filled with water and air, the sum of their volume fractions shall be 1 , and the constraints are as follows:

$$
\alpha_{a}+\alpha_{w}=1
$$

\section{Validation of results}

\subsection{Mesh independence analysis}

Under the same grid partition and encryption, the accuracy of hydrodynamic numerical calculation is positively correlated with the grid density in a certain range. The grid density is too small and the calculation accuracy is not enough; If the grid density is too large, the computing cost and time cost will be greatly increased. Therefore, before formal numerical calculation, it is necessary to conduct grid independence analysis ${ }^{[8]}$ to determine the appropriate grid size and quantity.

After determining the grid partition and encryption processing, take $0.1 \mathrm{~m}$ as the benchmark, select the refinement ratio of the grid in three directions according to the ITTC recommendation ${ }^{[9]}$, and set five grid foundation sizes respectively. The grid size and quantity are shown in Table 1.

Table 2. Grid size and quantity.

\begin{tabular}{lccccc}
\hline Grid size & 0.141 & 0.1 & 0.07 & 0.05 & 0.035 \\
\hline Grid quantity & 22.7 & 39.8 & 77.4 & 154.4 & 315.8 \\
\hline
\end{tabular}

Based on the above five grid densities, the resistance of SUBOFF is calculated under the condition of relative depth $\mathrm{H}^{\prime}=0.15\left(H^{\prime}=H / \mathrm{L}\right)$ and speed $V=2.288 \mathrm{~m} / \mathrm{s}$. The calculation results are shown in Figure 4.

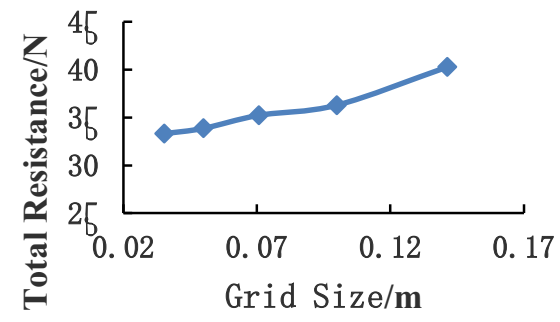

Fig. 4. Variation of total resistance with grid size.

According to the calculation results, the total resistance decreases monotonically with the decrease of grid size, and the total resistance values corresponding to $0.05 \mathrm{~m}$ and $0.0354 \mathrm{~m}$ are quite close. Therefore, it can be considered that the grid size of $0.05 \mathrm{~m}$ meets 
the requirements of grid density. However, it is necessary to compare the calculated value with the experimental value under the grid density to further verify the calculation accuracy.

\subsection{Calculation accuracy verification}

In this study, when the grid size is $0.05 \mathrm{~m}$ and the speed $V=2.288 \mathrm{~m} / \mathrm{s}$, the total resistance at SUBOFF relative depth $H^{\prime}=0.15,0.2,0.25,0.3$ and 0.35 is calculated respectively. The comparison results between the calculated value of resistance coefficient and the experimental value are shown in Figure 5.

It can be seen from Figure 5 that the difference between the calculated value of the total resistance coefficient and the test value under each diving depth is less than 5\%, indicating that the calculation accuracy meets the requirements, and the subsequent resistance calculation can be carried out based on the calculation model.

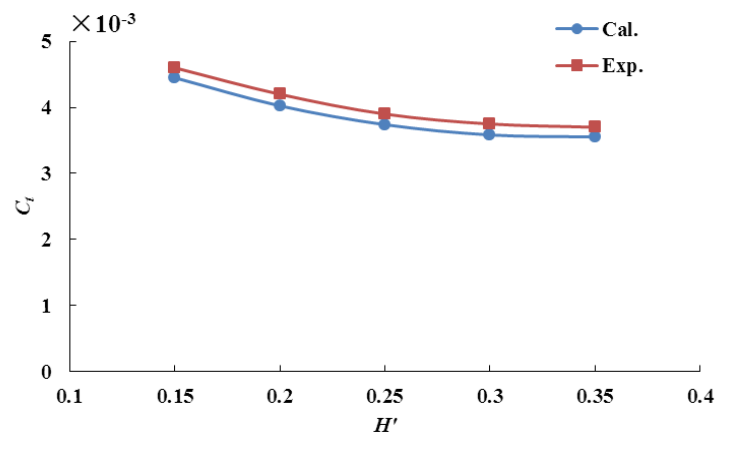

Fig. 5. Variation of total resistance coefficient with diving depth.

\section{Calculation conditions and result analysis}

\subsection{Calculation conditions}

In order to explore the influence of submergence and speed on the resistance of submarine sailing near the water surface, the resistance calculation of $V=2 \mathrm{Kn}, 4 \mathrm{kn}, 6 \mathrm{kn}, 8 \mathrm{kN}$ and $10 \mathrm{kN}$ is carried out for SUBOFF under relative submergence $H^{\prime}=0.15,0.2,0.25,0.3$ and 0.35 respectively, and the total resistance $R_{T}$, pressure resistance $R_{P}$ and friction resistance $R_{f}$ under various working conditions are calculated. The dimensionless treatment is carried out for each resistance component to obtain the dimensionless resistance coefficient:

$$
\begin{aligned}
& R_{T}{ }^{\prime}=\frac{R_{T}}{\frac{1}{2} \rho V^{2} L^{2}} \\
& R_{P}{ }^{\prime}=\frac{R_{P}}{\frac{1}{2} \rho V^{2} L^{2}} \\
& R_{f}{ }^{\prime}=\frac{R_{f}}{\frac{1}{2} \rho V^{2} L^{2}}
\end{aligned}
$$


The Froude coefficient $F r$ obtained by dimensionless treatment of speed $V$ is:

$$
F r=\frac{V}{\sqrt{g L}}
$$

In the above formula, $\rho$ is the sea water density, $g$ is the gravitational acceleration, $V$ is the speed, and $\mathrm{L}$ is the hull length.

\subsection{Result analysis}

\subsubsection{Calculation results of friction resistance coefficient}

Figure. 6 and Figure. 7 respectively reflect the variation of friction resistance coefficient $R_{f}$ ' with depth of submergence and speed. It can be seen that when $F r \leq 0.63, R_{f}$ ' gradually decreases with the increase of depth of submergence, and when $F r=0.787, R_{f}^{\prime}$ gradually increases with the increase of depth of submergence, but the overall variation range is very small; At all depths, $R_{f}{ }^{\prime}$ decreases with the increase of speed, and the reduction speed is basically the same.

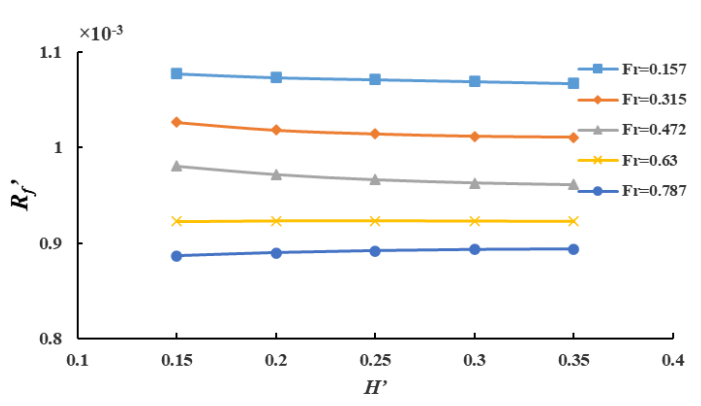

Fig. 6. Variation of friction coefficient with diving depth.

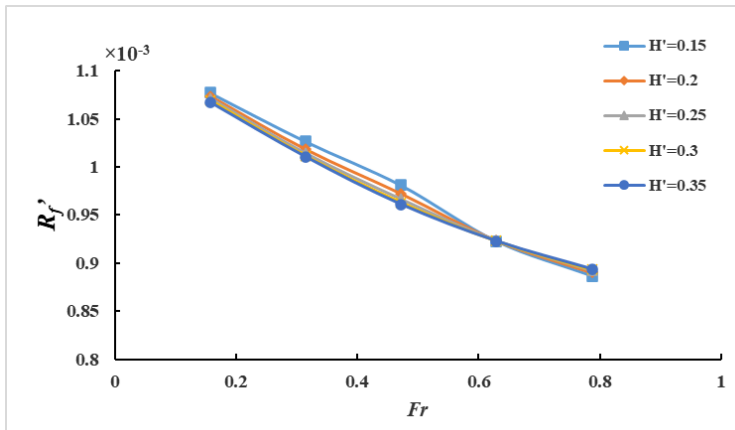

Fig. 7. Variation of friction coefficient with speed.

According to 1957ITTC plate friction coefficient formula:

$$
C_{f}=\frac{0.075}{(\lg \mathrm{Re}-2)^{2}}
$$

It can be seen that the plate friction resistance coefficient $C_{f}$ is related to the Reynolds number $R e$, and the formula of $R e$ is as follows: 


$$
R_{e}=\frac{V L}{V}
$$

where, $v$ Is the kinematic viscosity coefficient of water.

Therefore, at a fixed speed and hull length, the size of $C_{f}$ is the same as $v$ Relevant, and $v$ Related to pressure, the depth of submergence will affect the pressure, so the depth of submergence will affect the friction resistance coefficient $C_{f}$ of the plate.

$C_{f}$ can also be expressed as:

$$
C_{f}=\frac{R_{f}}{\frac{1}{2} \rho V^{2} S}
$$

where, $S$ is the wet surface area of the hull.

Similarly, the depth of submergence will also affect the dimensionless friction resistance coefficient $R_{f}$ '. The above formula also explains why $R_{f}$ ' decreases with the increase of speed.

\subsubsection{Calculation results of pressure resistance coefficient}

Figure. 8 and Figure. 9 respectively reflect the variation of pressure drag coefficient $R_{P}{ }^{\prime}$ with depth of submergence and speed. It can be seen that at all speeds, $R_{P}{ }^{\prime}$ decreases gradually with the increase of depth of submergence; Under each depth of submergence, the overall change trend of $R_{P}{ }^{\prime}$ with the increase of speed is that it first increases and then decreases, and the inflection point gradually moves backward with the increase of depth of submergence.

For submarines sailing near the water surface, the pressure resistance consists of wave making resistance and viscous pressure resistance. The wave making resistance mainly comes from the free liquid surface. With the increase of diving depth, the disturbance of the free liquid to the submarine gradually decreases, and the wave making resistance also gradually decreases. The viscous pressure resistance is mainly related to the shape of the hull, but not to the depth and speed. Therefore, the pressure resistance decreases gradually. Because the wave making resistance is approximately proportional to the 6th power of the speed and the viscous pressure resistance is approximately proportional to the square of the speed, the pressure resistance coefficient $R_{P}{ }^{\prime}$ decreases gradually with the increase of the diving depth.

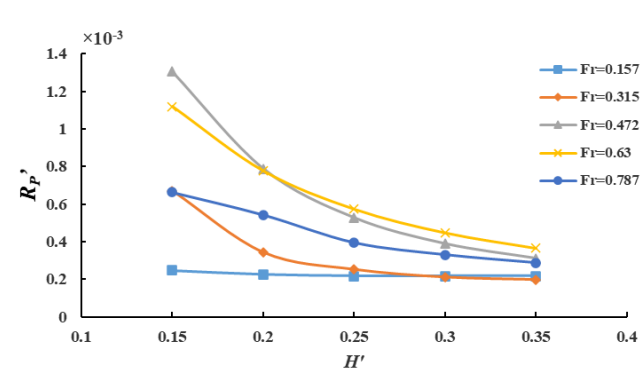

Fig. 8. Variation of pressure resistance coefficient with diving depth. 


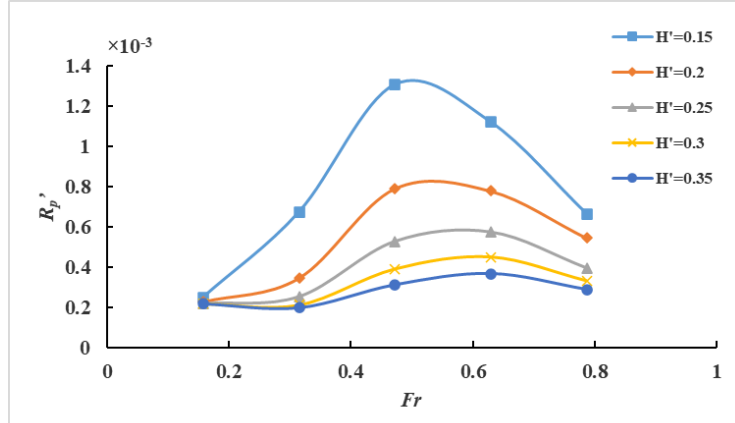

Fig. 9. Variation of pressure resistance coefficient with speed.

Figure 10 shows the relationship between wave making resistance coefficient $\mathrm{CW}$ and speed of different ship types ${ }^{[10]}$. Since the submarine belongs to an extremely thin ship type, its $\mathrm{CW}$ increases first and then decreases with the increase of $F r$, and the peak appears near $F r=0.55$. Since the viscous pressure resistance coefficient is basically unchanged, this explains the reason why the dimensionless pressure resistance coefficient $R_{P}{ }^{\prime}$ first increases and then decreases with the increase of speed, and the peak value of the curve of $R_{P}{ }^{\prime}$ with $F r$ under each diving depth is mainly near $F r=0.55$.

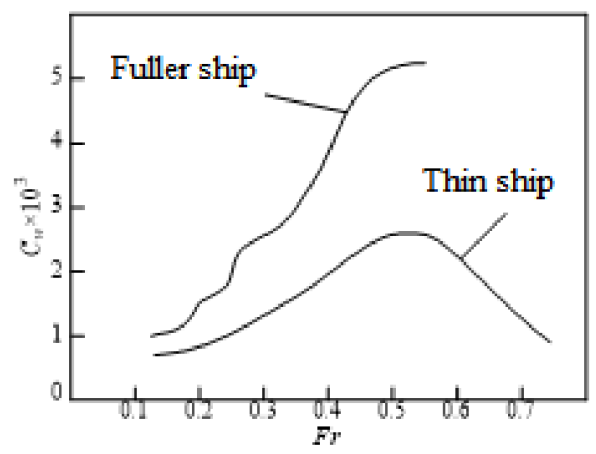

Fig. 10. Wave resistance coefficient curve.

\subsubsection{Calculation results of total resistance coefficient}

Figure.11 and Figure.12 respectively show the variation of total resistance coefficient $R_{T}{ }^{\prime}$ with submergence and speed. Since $R_{t}{ }^{\prime}=R_{f}{ }^{\prime}+R_{P}{ }^{\prime}$ and speed, the variation of $R_{T}{ }^{\prime}$ with submergence and speed is the superposition of $R_{f}{ }^{\prime}$ and $R_{P}{ }^{\prime}$ with submergence and speed. It can be seen from the results that the variation trend of $R_{T}{ }^{\prime}$ with diving depth and speed is basically consistent with $R_{P}{ }^{\prime}$. This is because the friction resistance coefficient $R_{f}^{\prime}$ of submarines is generally small and the variation range is small, and the pressure resistance coefficient RP' occupies the main position in $R_{T}{ }^{\prime}$. Therefore, $R_{P}{ }^{\prime}$ determines the variation trend of $R_{T}{ }^{\prime}$ with diving depth and speed. According to the calculation results in 5.2.2, $R_{P}{ }^{\prime}$ is mainly affected by the free surface, so $R_{T}{ }^{\prime}$ is greatly affected by the free surface. The results also show that the free surface has a great influence on the total resistance of submarines sailing near the water surface, which should be paid attention to. 


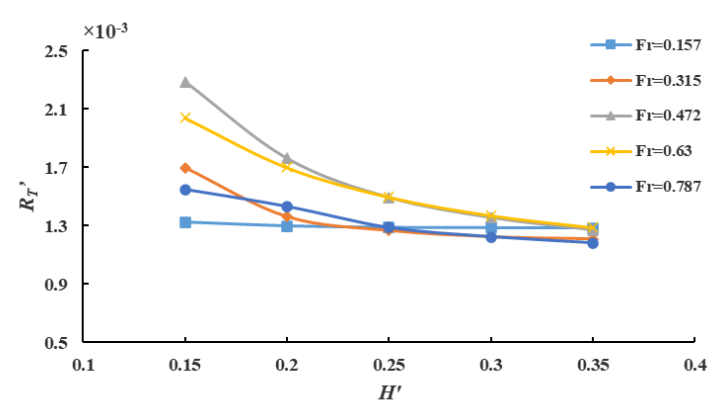

Fig. 11. Variation of total resistance coefficient with diving depth.

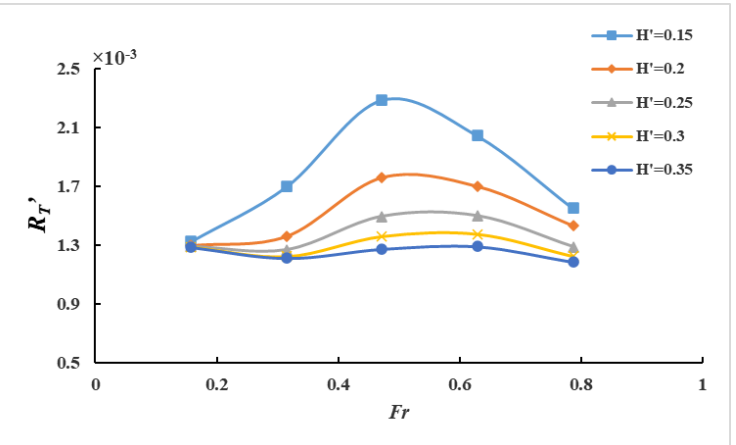

Fig. 12.Variation of total resistance coefficient with speed.

\subsubsection{Waveform analysis}

\section{(1) Influence of depth of submergence on waveform}

It can be seen from the results in 5.2.3 that the total resistance coefficient gradually decreases with the increase of diving depth. In order to intuitively reflect the influence of diving depth on the free surface waveform, Figure 13 shows the free surface wave height diagram under each diving depth when $\mathrm{fr}=0.472$. It can be seen that with the gradual increase of diving depth, the absolute values of peak value and trough value of free surface gradually decrease, indicating that the mutual interference between hull and free surface gradually attenuates.

The wave making of the free surface under each diving depth constitutes the Kelvin wave system, which includes shear wave and scattered wave. With the gradual increase of diving depth, the effect of shear wave is more significant and the effect of scattered wave is gradually weakened. This is of great significance for the study of submarine concealment.

\section{(2) Influence of speed on wave making}

According to the results in 5.2.3, the total resistance coefficient first increases and then decreases with the increase of speed. In order to reflect the influence of speed on free surface wave making, Figure 14 shows the free surface wave making diagram at each speed when $H^{\prime}=0.15$. It can be seen that with the increase of the speed, the amplitude of the rising wave first increases and then decreases. Thus, it intuitively reflects that under the same diving depth, with the increase of speed, the mutual interference effect between free surface and hull first increases and then decreases. 


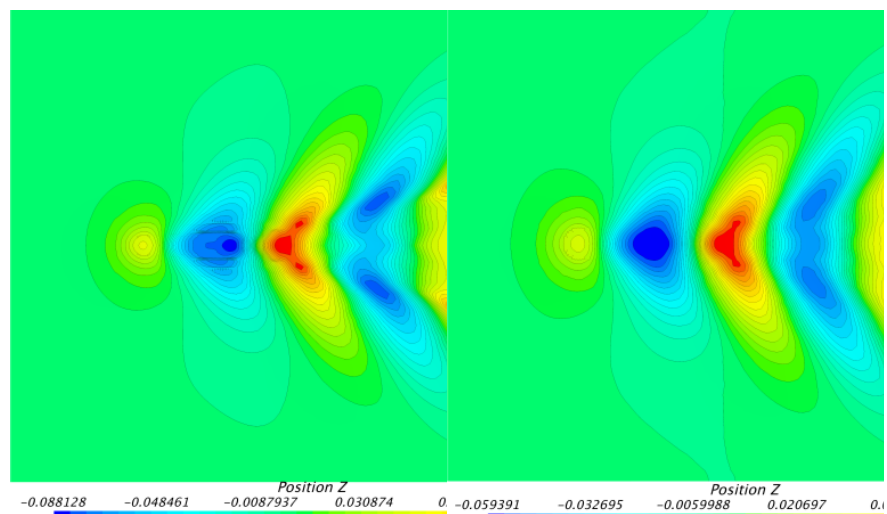

(a) $H^{\prime}=0.15$

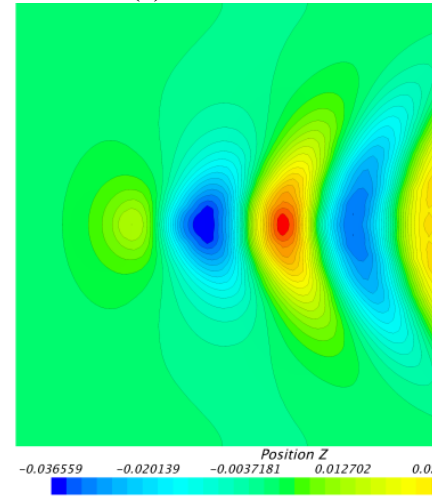

(d) $H^{\prime}=0.3$

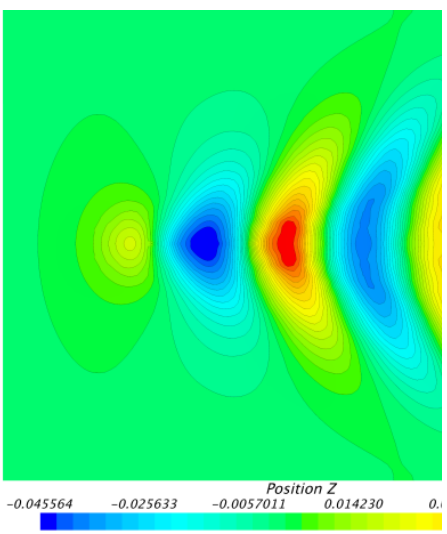

(c) $H^{\prime}=0.25$

Fig. 13. Waveform of free surface under each diving depth $(F r=0.472)$.

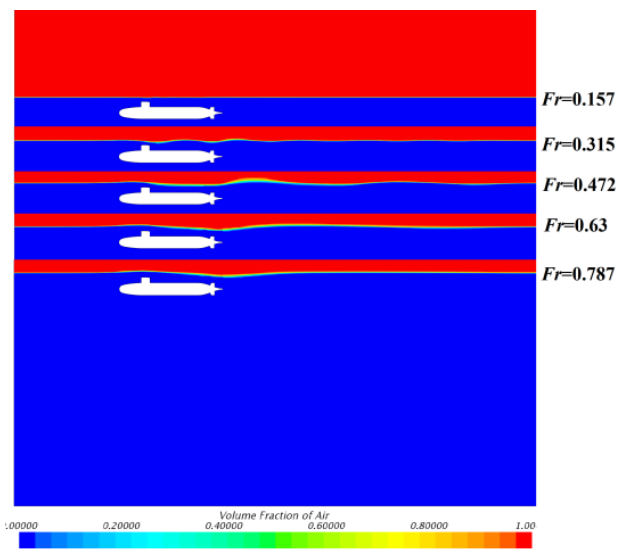

Fig. 14. Free surface wave making diagram at various speeds $\left(H^{\prime}=0.15\right)$. 


\section{Conclusion}

Taking SUBOFF with appendage as the research object, this paper carries out the resistance calculation under various depths and speeds, discusses the influence of depths and speeds on various resistance coefficients, and explores the changes of interference effects of free surface and hull under variable depths and speeds through waveform analysis. The conclusions are as follows:

(1) For the submarine sailing near the water surface, the free surface rises waves and generates wave making resistance mainly through the mutual interference with the hull, which affects its resistance characteristics;

(2) The depth of submergence has little effect on the coefficient of frictional resistance $R_{f}$ ' and great effect on the coefficient of compressive resistance $R_{P}{ }^{\prime} . R_{P}$ ' generally decreases with the increase of depth of submergence. $R_{P}$ ' determines the change trend of the total resistance coefficient $R_{T}$ 'and the two are basically the same;

(3) With the increase of speed, $R_{f}$ ' decreases gradually, and the overall change trend of $R_{P}$ ' increases first and then decreases, and the peak value of $R_{P}$ ' under each diving depth is near $F r=0.55$, and the change trend of $R_{T}$ ' is consistent with $R_{P}{ }^{\prime}$;

(4)With the increase of diving depth, the mutual interference between the hull and the free surface gradually attenuates, the shear wave of Kelvin wave system is more significant, and the scattered wave effect is weakened, which is of great significance for the study of submarine concealment; With the increase of the speed, the interaction effect between the free surface and the hull first increases and then decreases; Both of them confirm the calculation results of the above resistance coefficient.

\section{References}

1. ZHANG N, SHEN H C, YAO H Z. Numerical simulation of flow around submarine operating close to the bottom or near surface[J]. Journal of Ship and Mechanics, 2007, 11(4): 498-507.

2. ZHANG N, ZHANG S L. Numerical simulation of hull/propeller interaction of submarine in s-ubmergence and near surface conditions[J]. Journal of Hydrodynamics, 2014, 26(1): 50-56.

3. LI J.Numerical Simulation of Some Hydrodynamic Forces for Submerged Body Advancing Ne-ar Free Surface[D].Harbin: Harbin Engineering University, 2007.

4. QIU Y M, DENG R. Investigation on the Characteristics of the Wave Induced by a Near Free Surface Submarine[J]. Ship Science and Technology, 2016, 38(23): 44-47.

5. WILSON H S. An Investigation into the Wave-making Resistance of a Submarine Travelling Below the Free Surface[D]. Launceston Australia: National Centre of Maritime .

6. LI S Q, XIAO C R, CAO Z J. Numerical Analysis of Wake Flow and Hydrodynamics for a Su-bmarine Based on STAR-CCM+[J]. Chinese Journal of Ship Research, 2018, 13(Supp 1): 29-35.

7. YANG Q,WANG G D,ZHANG Z G,et al.Numerical Simulation of the Submarine Self-P-ropulsion Model Based on CFD Technology[J]. Chinese Journal of Ship Research, 2013, 8(2):22-27.

8. BAI T C, XU J, WANG G D, et al. Analysis of Resistance and Flow Field of Submarine Sailing Near the Ice Surface[J]. Chinese Journal of Ship Research, 2021, 16(2): $1-13$. 
9. ZHANG N,SHEN H C,YAO H Z.Uncertainty Analysis in CFD for Resistance and Flow Field[J]. Journal of Ship Mechanics,2008,12(002):211-224.

10. SHENG Z B,LIU Y Z.Ship Principle [M]. Shanghai Jiaotong University Press, 2004. 